\title{
Architecting
}

\section{the Digital Enterprise}

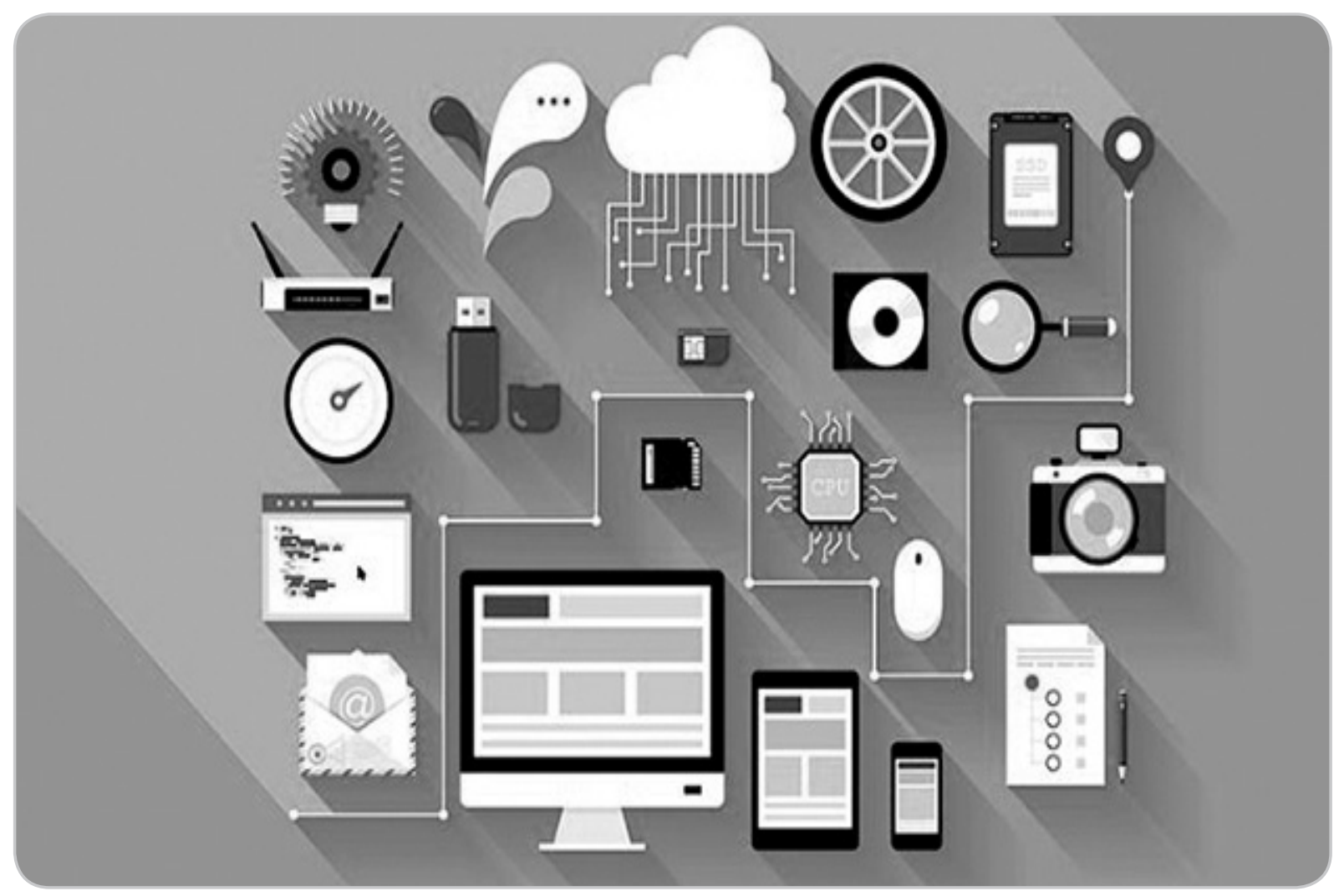

A survey of almost 4,500 IT leaders in 90 countries shows that the fastest-growing area of demand in IT this year is for enterprise architects. It is not a skills shortage, but an adoption lag.

The predominant mode of IT delivery over the past 30 years has been for structured, waterfall implementations at scale. Many domain areas have experienced consolidation of software into a few, large, ubiquitous products: the dominance of SAP, Oracle and Microsoft. The next 30 years will not be the same - not even the next three years.

This guide showcases some of the men who are helping women to progress in the technology industry and the methods they are using to do so.

In the historic mode of delivery, enterprise architects started at the top of the $\mathrm{V}$ model, translating business needs into a technology strategy, requirements and then to architectures. Failure in this task would be costly: time must be taken, and risk avoided. Architecture frameworks, such as The Open Group Architecture Framework (TOGAF), prized thoroughness - as did programme assurance frameworks such as CMMi. Architects were long-distance runners.

This is not the world we live in today. Businesses demand pace. They need to get capabilities to market quickly, and risk is to be managed rather than avoided. Failure is tolerable if it is fast. The $\mathrm{V}$ model is now an agile circle, and architects must now sprint.

This year's Harvey Nash/KPMG ClO survey shows the fastest-growing skills shortage in IT is for enterprise architects, up $26 \%$. This is not a skills shortage, 
but an adaptation lag - designing and congiguring digital platforms demands a new architecture mentality.

Enterprise architects remain paramount in delivering robust, effective IT that meets the needs of the business. But the enterprise architect mindset needs to evolve. Too many organisations tell us their architecture functions manage compliance processes instead of meeting business needs: that they are slow, are blockers or are bypassed for the most urgent demands.

Enterprise architects need to respond to this, and organisations need to consider their architecture o perating model and culture. To be nimble requires an organisation to empower those a rchitects closest to the business needs - those with domain expertise. To maintain consistency amidst this new autonomy, an enterprise's domain architects need to operate with a consensus around the approach to key architecture "plays" such as cloud, security and analytics.

The enterprise architect of the future needs to be able to grasp and manage risk: understanding what to solve now and what to solve iteratively. As the dominance of the biggest players has eroded, they must construct fluid ecosystems of software, where a product may be used to deliver a business out- come for one or two years until enterprise toolsets evolve. This is a different mentality for architects one which tolerates risk and even sprawl so long as it is managed and iteratively resolved.

Organisations are responding to growing political and economic uncertainty over the past 12 months by spending more on innovative, quickly adaptable technologies.

As digital transformation is essentially technology-enabled and driven innovation, there is a real opportunity for ClOs to be out in front helping their businesses lead the agenda.

There are many people in IT who operate in this way - and who don't call themselves enterprise architects. So perhaps the shortage of enterprise architects reflects a failure; both on the part of existing enterprise architects acting this way, and in the lag of organisations adapting to the new paradigm.

But it would be an equal failure not to see the value of combining traditional enterprise architect virtues - an intimate understanding of the business, a forward-looking view of technology and a robust understanding of delivery - with these newer and nimbler architecture skills. This reset may not endure another full 30 years unchanged - but it's an effective model for the digital world. 